\title{
Validation of the Thorax Trauma Severity Score for mortality and its value for the development of acute respiratory distress syndrome
}

This article was published in the following Dove Press journal:

Open Access Emergency Medicine

22 August 2011

Number of times this article has been viewed

\author{
Tjeerd S Aukema' \\ Ludo FM Beenen² \\ Falco Hietbrink' \\ Luke $\mathrm{PH}$ Leenen' \\ 'Department of Surgery, University \\ Medical Center Utrecht, Utrecht, \\ ${ }^{2}$ Department of Radiology, Academic \\ Medical Center, Amsterdam, \\ The Netherlands
}

Background: The aim of the present study was to evaluate and to validate the Thorax Trauma Severity Score (TTSS) for mortality.

Methods: By database analysis 712 patients with an injury to the chest admitted to the Universal Medical Center Utrecht between 2000 and 2004 were studied. All patients with a score of $\geq 1$ on the AIS thorax were included in the study. The patients' file was evaluated for: TTSS, intensive care unit stay, days on ventilation, thorax trauma-related complications (eg, acute respiratory distress syndrome [ARDS]), total hospital stay, and mortality.

Results: Of the 516 patients included in the study, 140 (27\%) developed thorax-related complications. The overall in-hospital mortality rate was $10 \%$. The receiver operating characteristic curve for predicting mortality demonstrated an adequate discrimination by a value of 0.844 . The TTSS was statistically significant higher in patients who died of thorax-related complications than in patients who died because of nonthorax-related complications and survivors $(P<0.001$, confidence interval [CI] 95\%). In patients who developed ARDS the TTSS was significant higher $(P=0.005$, CI 95\%).

Conclusion: This study supports the use of the TTSS for predicting mortality in thoracic injury patients. Furthermore, the TTSS appears capable of predicting ARDS.

Keywords: wounds and injuries, thorax, trauma severity indices, acute respiratory distress syndrome, mortality

\section{Introduction}

Rapid and accurate assessment of the chest in blunt trauma patients is important to direct life-saving and definitive management. Solid risk stratification of thoracic trauma is needed for individual management of ventilation, intensive care support, and surgical strategy selection to reduce morbidity and mortality. If patients are not treated properly they can suffer from major disabilities. ${ }^{1,2}$

The present standards for assessing thoracic trauma vary widely. ${ }^{3-5}$ A scoring system that can help predict thorax related complications in thoracic trauma patients is needed. For this in 2000 Pape et $\mathrm{al}^{6}$ developed the Thorax Trauma Severity Score (TTSS). As demonstrated in Table 1, the TTSS combines the patient's age, resuscitation parameters, and radiological assessment of the thorax. After the first publication in 2000 the score has never been validated by any other independent study, and the association of the score with thoracic related death and mortality has not been explored. This makes it more difficult to add it to other widely used scorings systems, such as the Glasgow Coma Score $^{7}$ or the Injury Severity Score. ${ }^{8}$
Department of Surgery, G04.228, University Medical Center Utrecht, PO Box 85500, 3508 GA Utrecht,

The Netherlands

Tel +3 I 887559882

$\mathrm{Fax}+31302541944$

Email Ileenen@umcutrecht.nl 
Table I The TTSS developed by Pape et a $^{6}$ to predict mortality in thoracic trauma patients

\begin{tabular}{lllllll}
\hline Grade & $\mathbf{P a O}_{2} / \mathrm{FiO}_{2}$ & Rib fracture & Contusion & Pleural involvement & Age & Points \\
\hline 0 & $>400$ & 0 & None & None & $<30$ & 0 \\
I & $300-400$ & I-3 & I lobe, unilateral & PT & $30-4$ I & I \\
II & $200-300$ & $3-6$ & I lobe, bilateral or & HT/HPT unilateral & $42-54$ & 2 \\
& & & 2 lobes unilateral & & $55-70$ & 3 \\
II & $150-200$ & $>3$ bilateral & $<2$ lobes bilateral & HT/HPT bilateral & $>70$ \\
IV & $<$ I50 & Flail chest & $\geq 2$ lobes bilateral & TPT & 5 \\
\hline
\end{tabular}

Notes: For calculation of the total score, all categories are summed. A minimum value of 0 points and a maximum value of 25 points can be achieved.

Abbreviations: PT, pneumothorax; HT, hemothorax; HPT, hemopneumothorax; TPT, tension pneumothorax; TTSS, Thorax Trauma Severity Score.

The aim of the present study was to evaluate and to validate the TTSS for mortality and evaluate the correlation between the TTSS and thorax-related complications such as acute respiratory distress syndrome (ARDS).

\section{Materials and methods}

All patients admitted to the University Medical Center Utrecht level-1 emergency department (ED) from January 1, 2000 until January 1, 2005 were evaluated. We searched the trauma database for patients with any injury to the chest and performed a retrospective analysis. Written informed consent was not required because of the retrospective nature of the investigation.

The TTSS employs 5 specific parameters: rib fractures, lung contusion, $\mathrm{PaO}_{2} / \mathrm{FiO}_{2}$ ratio, age, and pleural involvement. These parameters are coded from 0 to 5 . The TTSS is calculated by adding the coded values for each of these five parameters; minimum score is 0 and maximum score is 25 . The individual TTSS was calculated for every patient (see Figure 1).

Patients were included in this study if they scored $\geq 1$ on the Abbreviated Injury Score $_{\text {thorax }}\left(\right.$ AIS $\left._{\text {thorax }}\right){ }^{9}$ Patients referred from other hospitals were included if the referral

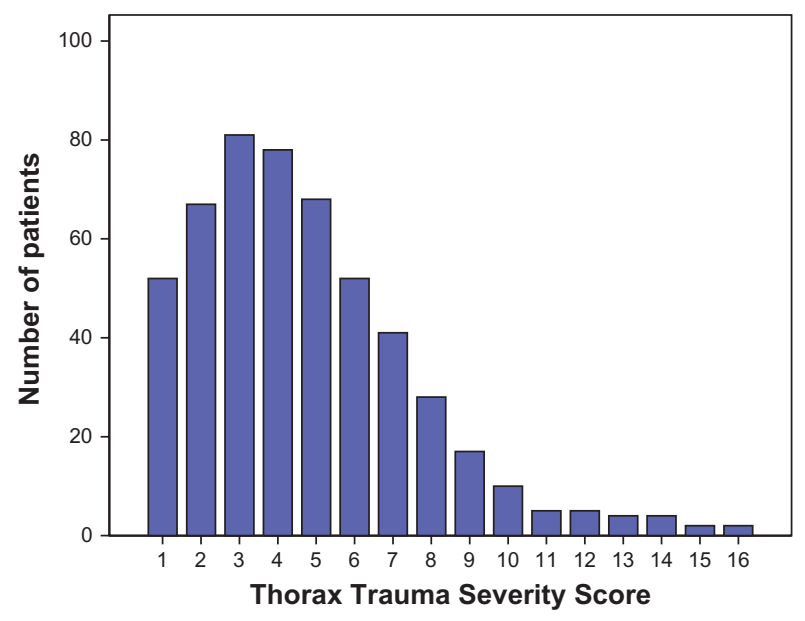

Figure I Distribution of the number of patients and the Thorax Trauma Severity Score in 516 thorax trauma patients. was within 8 hours and if major surgical procedures were done in our institution.

The following factors were retrieved from the original patients' files: TTSS parameters as mentioned above, patients' age, sex, base excess at admission, total hospital stay, intensive care unit stay, resuscitation length, thorax-related complications, and mortality. The admission day chest X-rays of every patient were independently evaluated by a trauma radiologist (LB). The following thorax-related complications were registered: pneumonia, second pneumothorax, persistent hematothorax, ARDS, ${ }^{10}$ and empyema.

\section{Statistical analysis}

Statistical analyses were performed by using SPSS 15 (v 15, for Windows, SPSS, Inc, Chicago, IL). The association between various parameters was evaluated using unvaried analysis. Statistical analysis was performed with the nonparametric MannWhitney $U$ test to compare two groups and Kruskall-Wallis $H$ test to compare multiple groups. Predictive values were calculated using a receiver operating characteristic curve (ROC) analysis. Statistical significance was defined as $P<0.05$.

\section{Results \\ Demographics}

Of the 712 patients in total registered in the study period with an injury to the chest, 189 did not score any point on the AIS $_{\text {thorax }}$ and were excluded. This excluded group harbored for example patients with a minor laceration to the chest wall. So, in total 516 patients $(73 \%)$ were included in the study. Demographic data are presented in Table 2.

\section{Admission severity and events}

Of TTSS characteristics, $70 \%$ of the 516 included patients at least one rib fracture (Table 3). Lung contusion was diagnosed in $44 \%$, pneumothorax in $26 \%$, hemothorax in $13 \%$, and tension pneumothorax in $2 \%$.

The distribution patten in our study cohort among the different TTSS scores was almost normal (Figure 1). 
Table 2 Patient demographics

\begin{tabular}{ll}
\hline Number of patients & 516 \\
Mean age (years) & 43 \\
Sex of patient, $n$ (\%) & \\
$\quad$ Male & $375(73 \%)$ \\
$\quad$ Female & $141(29 \%)$ \\
Days of hospital stay (median) & 23 \\
Patients in ICU, $\mathrm{n}(\%)$ & 271 (53\%) \\
Length of ICU stay (median) & 8 \\
Ventilated patients, $\mathrm{n}(\%)$ & $233(45 \%)$ \\
Length of ventilation (median days) & 7 \\
TR complications, $\mathrm{n}(\%):$ & $140(27)$ \\
Pneumonia & $102(20 \%)$ \\
Second pneumothorax & $23(5 \%)$ \\
Second hematothorax & $9(2 \%)$ \\
Empyema & $8(2 \%)$ \\
ARDS & $29(6 \%)$ \\
Total patients & $140(27 \%)$ \\
Mortality & $52(10 \%)$ \\
\hline
\end{tabular}

Abbreviations: ICU, intensive care unit; n, number; TR, thorax-related; ARDS, acute respiratory distress syndrome.

As expected, the peak number of patients was within the lower TTSS scores, most patients scoring three points (modus). The maximum score of patients admitted alive to our ED was 16.

The overall in-hospital mortality rate was $10 \%$ (53 patients). Mortality was correlated with high TTSS. The ROC was used to demonstrate the sensitivity and specificity of the TTSS for predicting mortality during hospital stay. The area under the curve (AUC) shows a value of 0.844 (Figure 2). Patients who died of thoracic-related complications had a higher TTSS than patients who survived ( $P \leq 0.001$, CI 95\%) (Figure 3). Patients who died of thoraxrelated complications scored higher than patients who died

Table 3 Patients chest-wall injuries of the TTSS

\begin{tabular}{ll}
\hline Chest-wall injuries & Incidence n (\%) \\
\hline Rib fractures & $363(70 \%)$ \\
Unilateral & $325(90 \%)$ \\
Bilateral & $38(10 \%)$ \\
Lung contusion & $230(44 \%)$ \\
Unilateral & $145(63 \%)$ \\
Bilateral & $85(37 \%)$ \\
Pneumothorax & $137(26 \%)$ \\
Unilateral & $126(92 \%)$ \\
Bilateral & $11(8 \%)$ \\
Hemothorax & $68(13)$ \\
Unilateral & $65(96 \%)$ \\
Bilateral & $3(4 \%)$ \\
Tension pneumothorax & $12(2 \%)$ \\
Hemopneumothorax & $19(4 \%)$ \\
Unilateral & $17(89 \%)$ \\
Bilateral & $2(11 \%)$ \\
\hline
\end{tabular}

Abbreviations: $n$, number; TTSS, Thorax Trauma Severity Score.

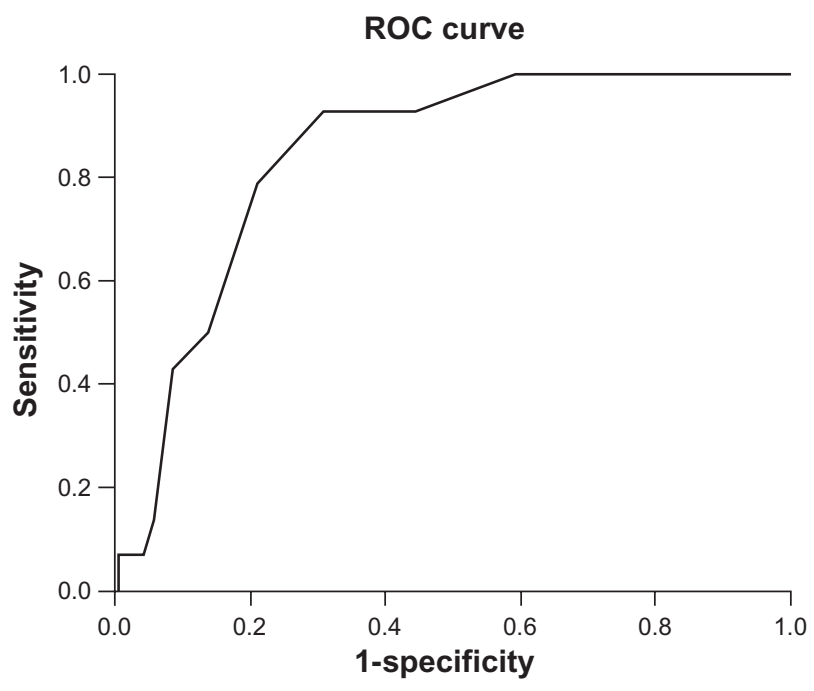

Figure 2 Receiver operating characteristic curve (ROC) analysis of the Thorax Trauma Severity Score, designed to predict mortality. The area under the curve shows a value of 0.844 .

of nonthorax-related complications $(P=0.014$, confidence interval 95\% [CI 95\%]). No significant difference was found between the group of patients who died of nonthorax-related causes and the surviving patients $(P=0.114$, CI 95\%).

The total number of thorax trauma-related complications was 140 (27\%): 102 patients suffered from hospital-acquired pneumonia, 23 patients developed a secondary pneumothorax, nine patients developed a persistent hematothorax, empyema was seen in eight patients, and 29 patients were diagnosed with ARDS (Table 2).

Patients who developed ARDS scored a higher TTSS than non-ARDS patients $(P=0.005$, CI 95\%) (Figure 4). Of note, four patients who scored 15 or 16 points did not

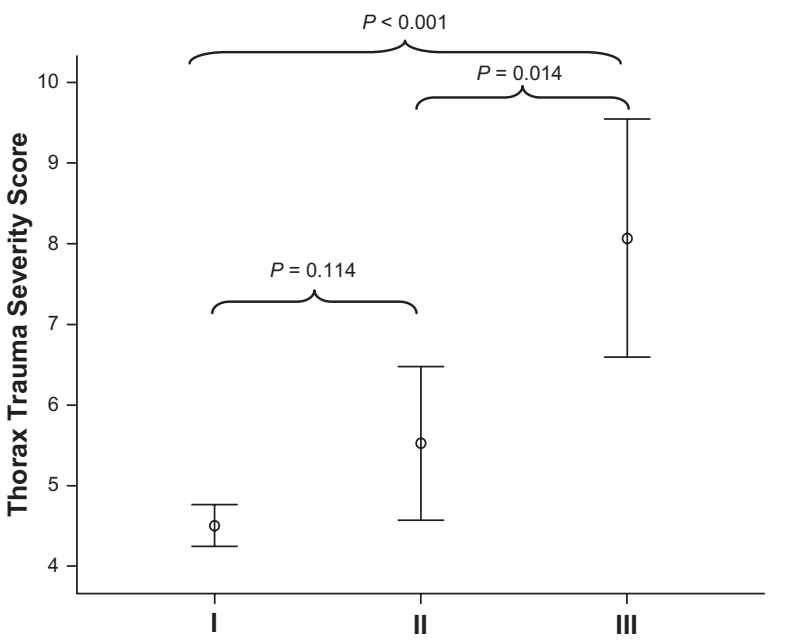

Figure 3 A standard error of mean figure of the Thorax Trauma Severity Score and patients who survived (I), patients who died of nonthorax-related complications (II), and patients who died of thorax-related complications (III). 


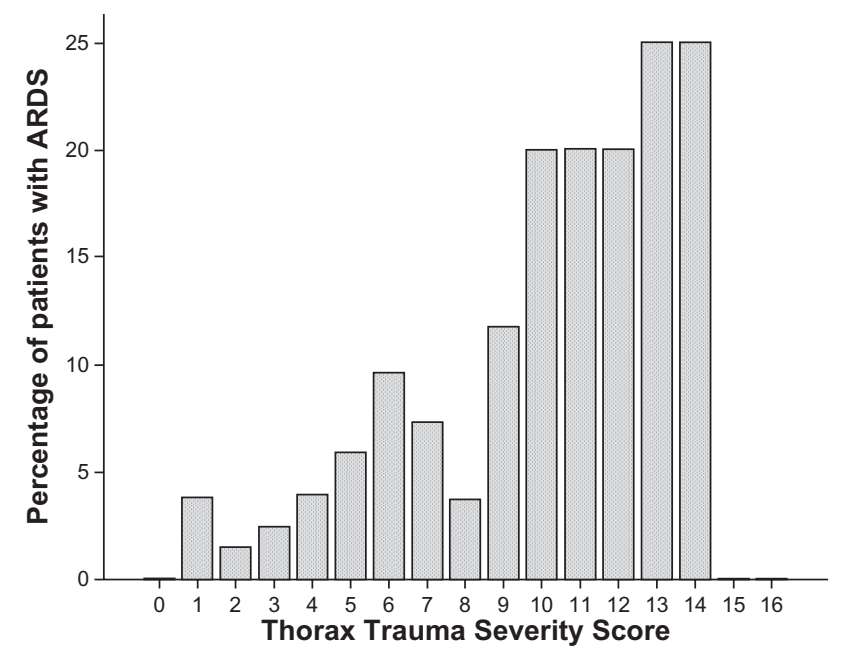

Figure 4 Percentage of patients with acute respiratory distress syndrome (ARDS) and Thorax Trauma Severity Score.

develop ARDS as they died earlier in the process and did not have the time to develop it.

\section{Discussion}

This is the first study to validate the TTSS for mortality and describe an association between the score and the development of ARDS.

We analyzed the collected data from original patients' files of a total number of 516 chest trauma patients. All radiological findings were reviewed by the same dedicated trauma radiologist, which ensured a consistent way of reviewing every chest X-ray and ruled out inter-observer variability occurring in radiological documentation reports.

We used the results from the admission chest X-ray, as originally described by Pape. ${ }^{6}$ This type of radiological diagnostics will probably still be used for an extensive time in the advanced trauma life support. ${ }^{11}$ This modality is easily available in every hospital. By using the chest $\mathrm{X}$-ray, the TTSS can also be used in hospitals where early chest computed tomography (CT) scan is not yet fully integrated in the early care. Moreover, the chest X-ray is a quick modality with a specific diagnostic yield and still is of importance in the early work up of trauma patients. To put it in perspective, however, chest X-ray can miss rib fractures more than $50 \%$ of the time compared to the CT scan.

The predictive value of the TTSS could not be demonstrated in this study since the method was not set up to calculate the predictive value of the TTSS. Nevertheless, we did demonstrate a clear association between the TTSS and several outcome parameters.
Several general scorings systems for trauma patients have shown excellent values for predicting outcome..$^{12,13}$ In concordance with trauma scores such as the New Injury Severity Score (AUC of 0.827$)^{14}$ the TTSS demonstrated a high AUC of 0.844 which makes it a sensitive and specific scoring system for predicting mortality. In addition our study was able to demonstrate an association between the TTSS and thorax-related death. The score was significantly higher than in patients who died of other complications and even more so in all patients who survived. This extra characteristic of the score could be of added value in trauma evaluation.

The thorax trauma-related complication rate has been shown to be high (27\%) and can be severe. ${ }^{15}$ Although the mortality of ARDS has declined during the last decade, ARDS is still is one of the most serious thorax trauma-related complications, with a mortality rate of $20 \%-43 \%,{ }^{16,17}$ and accounts for about $7 \%-9 \%$ of intensive care unit admissions per year. ${ }^{18}$ However, Davidson et al concluded that there was no difference in the long-term mortality rate for ARDS patients. ${ }^{19}$ Miller et $\mathrm{al}^{20}$ published several independent risk factors for developing ARDS, but the maximum AUC was 0.72 . We showed that the TTSS is significantly higher in patients who develop ARDS after thorax trauma. To our knowledge no other scoring system has demonstrated an association with the development of ARDS. This may lead to a different clinical policy on blood transfusion in patients with a high TTSS. Restrictive transfusion policies and ventilation strategies are advised for these patients because these measures are associated with a decreased incidence of ARDS. 21,22

Some limitations of our study should be acknowledged. First, the design of the study was retrospective. Although it had an advantage that every chest X-ray was assessed by the same radiologist, this is also a limitation, because we were therefore unable to calculate inter-observer variability for interpretation of the chest X-rays with kappa statistics. The interpretation of the chest X-ray by the radiologist could not be compared with a gold standard because not all patients received a subsequent chest CT-scan in the $\mathrm{ED}$, yet this is the way the original score was constructed.

In future an increasing number of EDs will be equipped with a CT scanner for quick radiological diagnosis. The CT scan is more sensitive and specific than the conventional chest X-ray for diagnosing the radiological items used in the TTSS (rib fracture and lung contusion). ${ }^{23-25}$ In future studies, radiological results of the CT scan may be used for the TTSS to further improve the sensitivity and specificity of the scoring system. 
In conclusion this study supports the use of the TTSS for predicting mortality in thoracic injury patients. Furthermore, the TTSS appears capable of predicting ARDS.

\section{Prior publication}

This paper was first presented in part at the Annual Congress of the Dutch Society of Surgery [Nederlandse Vereniging voor Heelkunde], May 11, 2007, Veldhoven, The Netherlands.

\section{Disclosure}

The authors declare no conflicts of interest in relation to this paper.

\section{References}

1. Pirente N, Blum C, Wortberg S, et al. Quality of life after multiple trauma: the effect of early onset psychotherapy on quality of life in trauma patients. Langenbecks Arch Surg. 2007;392(6):739-745.

2. Vles WJ, Steyerberg EW, Essink-Bot ML, van Beeck EF, Meeuwis JD, Leenen LP. Prevalence and determinants of disabilities and return to work after major trauma. J Trauma. 2005;58(1):126-135.

3. Chawda MN, Hildebrand F, Pape HC, Giannoudis PV. Predicting outcome after multiple trauma: which scoring system? Injury. 2004; 35(4):347-358.

4. Meredith JW, Evans G, Kilgo PD, et al. A comparison of the abilities of nine scoring algorithms in predicting mortality. J Trauma. 2002; 53(4):621-628.

5. Wutzler S, Wafaisade A, Maegele M, et al. Lung Organ Failure Score (LOFS): Probability of severe pulmonary organ failure after multiple injuries including chest trauma. Injury. 2011 Jan 20. [Epub ahead of print].

6. Pape HC, Remmers D, Rice J, Ebisch M, Krettek C, Tscherne H. Appraisal of early evaluation of blunt chest trauma: development of a standardized scoring system for initial clinical decision making. J Trauma. 2000;49(3):496-504.

7. Teasdale G, Jennett B. Assessment of coma and impaired consciousness. A practical scale. Lancet. 1974;2:81-84.

8. Baker SP, O'Neill B, Haddon W Jr, Long WB. The injury severity score: a method for describing patients with multiple injuries and evaluating emergency care. J Trauma. 1974;14(7872):187-196.

9. Rating the severity of tissue damage. I. The abbreviated scale. JAMA. 1971;215(2):277-280.

10. Bernard GR, Artigas A, Brigham KL, et al. The American-European Consensus Conference on ARDS: definitions, mechanisms, relevant outcomes, and clinical trial coordination. Am J Respir Crit Care Med. 1994;149(3 Pt 1):818-824.
11. Kortbeek JB, Al Turki SA, Ali J, et al. Advanced trauma life support, 8th edition, the evidence for change. J Trauma. 2008;64(6):1638-1650.

12. Champion HR, Copes WS, Sacco WJ, et al. Improved predictions from a severity characterization of trauma (ASCOT) over Trauma and Injury Severity Score (TRISS): results of an independent evaluation. J Trauma. 1996;40(1):42-48.

13. Frankema SP, Steyerberg EW, Edwards MJ, van Vugt AB. Comparison of current injury scales for survival chance estimation: an evaluation comparing the predictive performance of the ISS, NISS, and AP scores in a Dutch local trauma registration. J Trauma. 2005;58(3):596-604.

14. Lavoie A, Moore L, LeSage N, Liberman M, Sampalis JS. The New Injury Severity Score: a more accurate predictor of in-hospital mortality than the Injury Severity Score. J Trauma. 2004;56(6):1312-1320.

15. Champion HR, Copes WS, Sacco WJ, et al. The Major Trauma Outcome Study: establishing national norms for trauma care. J Trauma. 1990;30(11):1356-1365.

16. Plurad D, Martin M, Green D, et al. The decreasing incidence of late posttraumatic acute respiratory distress syndrome: the potential role of lung protective ventilation and conservative transfusion practice. J Trauma. 2007;63(1):1-7.

17. Zambon M, Vincent JL. Mortality rates for patients with ALI/ARDS have decreased over time. Chest. 2008;133(5):1120-1127.

18. Wind J, Versteegt J, Twisk J, et al. Epidemiology of acute lung injury and acute respiratory distress syndrome in The Netherlands: a survey. Respir Med. 2007;101(10):2091-2098.

19. Davidson TA, Rubenfeld GD, Caldwell ES, Hudson LD, Steinberg KP. The effect of acute respiratory distress syndrome on long-term survival. Am J Respir Crit Care Med. 1999;160(6):1838-1842.

20. Miller PR, Croce MA, Kilgo PD, Scott J, Fabian TC. Acute respiratory distress syndrome in blunt trauma: identification of independent risk factors. Am Surg. 2002;68(10):845-850.

21. Gong MN, Thompson BT, Williams P, Pothier L, Boyce PD, Christiani DC. Clinical predictors of and mortality in acute respiratory distress syndrome: potential role of red cell transfusion. Crit Care Med. 2005;33(6): 1191-1198.

22. Toy P, Popovsky MA, Abraham E, et al. Transfusion-related acute lung injury: definition and review. Crit Care Med. 2005;33(4):721-726.

23. Reske AW, Reske AP, Heine T, et al. Computed tomographic assessment of lung weights in trauma patients with early posttraumatic lung dysfunction. Intensive Care Med. 2010 Nov;36(11):1836-1844.

24. Livingston DH, Shogan B, John P, Lavery RF. CT diagnosis of Rib fractures and the prediction of acute respiratory failure. $J$ Trauma. 2008;64(4):905-911.

25. Sangster GP, Gonzalez-Beicos A, Carbo AI, et al. Blunt traumatic injuries of the lung parenchyma, pleura, thoracic wall, and intrathoracic airways: multidetector computer tomography imaging findings. Emerg Radiol. 2007;14(5):297-310.
Open Access Emergency Medicine

\section{Publish your work in this journal}

Open Access Emergency Medicine is an international, peer-reviewed, open access journal publishing original research, reports, editorials, reviews and commentaries on all aspects of emergency medicine. The manuscript management system is completely online and includes a very quick and fair peer-review system, which is all easy to use.

\section{Dovepress}

Visit http://www.dovepress.com/testimonials.php to read real quotes from published authors. 